\title{
Guest Editorial Special Issue: High-Level Programming for Heterogeneous Parallel Systems
}

\author{
Christopher Brown ${ }^{1}$
}

High-Level Heterogeneous and Hierarchical Parallel Systems (HLPGPU) aims to bring together researchers and practitioners to present new results and ongoing work on those aspects of high-level programming relevant or specific to GPGPUs and new architectures. The 2017 HLPGPU symposium was an event co-located with the HiPEAC conference in Stockholm, Sweden. HLPGPU is targeted at high-level parallel techniques, including programming models, libraries and languages, algorithmic skeletons, refactoring tools and techniques for parallel patterns, tools and systems to aid parallel programming, heterogeneous computing, timing analysis and statistical performance models. The invited talk was given by Alastair Murray of Codeplay in Edinburgh. We had 9 paper presentations at the workshop, after which, we opened up the special issue for revised submissions.

This special issue of IJPP presents the following $\mathbf{8}$ accepted and revised papers.

- HitFlow: A Dataflow Programming Model for Hybrid Distributed- and Shared-Memory Systems Javier Fresno, Daniel Barba, Arturo GonzalezEscribano, Diego R. Llanos

- Efficient Dynamic Pinning of Parallelized Applications by Distributed Reinforcement Learning by Georgios C. Chasparis and Michael Rossbory

- Compiler Optimization of Accelerator Data Transfers by Matthew B. Ashcraft, Alexander Lemon, David A. Penry, Quinn Snell

- Verifying Parallel Code After Refactoring Using Equivalence Checking by Moria Abadi, Sharon Keidar-Barner, Dmitry Pidan, Tatyana Veksler

$\triangle$ Christopher Brown

cmb21@st-andrews.ac.uk

1 School of Computer Science, University of St Andrews, St Andrews, Fife KY16 9SX, Scotland, UK 
- The RePhrase Extended Pattern Set for Data Intensive Parallel Computing by Marco Danelutto, Tiziano De Matteis, Daniele De Sensi, Gabriele Mencagli, Massimo Torquati, Marco Aldinucci, Peter Kilpatrick

- Multi-device Controllers: A Library to Simplify Parallel Heterogeneous Programming by Ana Moreton-Fernandez, Arturo Gonzalez-Escribano, Diego R. Llanos

- Type-Driven Automated Program Transformations and Cost Modelling for Optimising Streaming Programs on FPGAs by Wim Vanderbauwhede, Syed Waqar Nabi, Cristian Urlea

- Parallel SIMD CPU and GPU Implementations of Berlekamp-Massey Algorithm and Its Error Correction Application by Hamidreza Mohebb

We thank all the speakers, the authors, the rest of the participants, the Program Committee and the IJPP SI editors for contributing to the success of HLPGPU 2017.

\section{HLPGPU Special Issue Editors}

- Marco Aldinucci (University of Torino, Italy)

- Jose Daniel Garcia (University Carlos III Madrid, Spain)

- Christopher Brown (University of St Andrews, UK)

\section{HLPGPU PC Members}

- Murray Cole (University of Edinburgh, UK)

- Marco Danelutto (University of Pisa, Italy)

- Jose Daniel Garcia (University of Carlos III Madrid, Spain)

- Arturo Gonzalez (University of Valladolid, Spain)

- Segei Gorlatch (University of Muenster, Germany)

- Gaetan Hains (Huawei)

- Vladimir Janjic (University of St Andrews, UK)

- Herbert Kuchen (University of Muenster, Germany)

- Zoltan Theisz (EvoPro, Hungary)

- Michael Vinov (IBM, Israel)

- Michael Wong (CodePlay, UK)

Open Access This article is distributed under the terms of the Creative Commons Attribution 4.0 International License (http://creativecommons.org/licenses/by/4.0/), which permits unrestricted use, distribution, and reproduction in any medium, provided you give appropriate credit to the original author(s) and the source, provide a link to the Creative Commons license, and indicate if changes were made. 